Walter WAGNER

Université de Vienne, Autriche

\title{
UN HOMME OBSCUR : LE TESTAMENT ÉCOLOGIQUE DE MARGUERITE YOURCENAR
}

Un homme obscur, «long récit ou roman court» ${ }^{1}$ selon la typologie yourcenarienne, fut publié en 1982 dans le recueil Comme l'eau qui coule et est considéré par l'auteur «comme une sorte de testament ${ }^{2}$. Ce texte d'une ampleur modeste ne constitue pas seulement le dernier ouvrage de fiction de Yourcenar, mais occupe également une place privilégiée au sein de son œuvre, dans la mesure où la nature s'impose dès le début de l'œuvre à la fois en tant que décor et actant. Le rôle que joue le souci de l'environnement dans la biographie et l'œuvre de l'écrivaine, en particulier Un homme obscur, est souligné dans une interview ${ }^{3}$ :

- La nature est importante pour vous?

- Naturellement. Pour nous tous.

- Elle est importante pour votre vie et pour votre œuvre?

- C'est plus important que tout. Nous sommes des objets dans la nature, c'est tout. [...]

- Et si vous deviez choisir une de vos auvres où vous auriez mis d'une manière particulière votre sentiment pour la nature?

- Pour la nature, pour la vie et pour l'homme ? Un Homme obscur. ${ }^{4}$

Une autre particularité du récit en question consiste dans le fait que la diégèse se déroule principalement sur des îles, lieu de prédilection de l'utopie (qui, en l'occurrence, porte l'empreinte d'un souci prononcé pour le monde naturel).

L'interview citée plus haut nous amène à énoncer l'hypothèse que Yourcenar a utilisé Un homme obscur pour faire passer des messages essentiels sur sa philosophie de la nature, supposition corroborée par la critique. Enrica Restori, par exemple, signale la déshumanisation subie par Nathanaël qu'elle qualifie d' "homo animalis » ${ }^{5}$. Lucile Desblache, quant à elle, insiste sur la « 'pensée naturelle' » ${ }^{6} \mathrm{du}$

\footnotetext{
1 YourCENAR Marguerite (1982), Postface d'Un homme obscur, Euvres romanesques, Paris, Gallimard, coll. de la Pléiade, p. 1065.

2 SAvigneau Josyane (2002), La Bienveillance singulière de Marguerite Yourcenar (1984), in: YourCenAR Marguerite, Portrait d'une voix. Vingt-trois entretiens (1952-1987), Paris, Gallimard, p. 324.

${ }^{3}$ Les écrits non fictionnels de Yourcenar (lettres, essais, notes, entretiens et jusqu'à ses mémoires Le Labyrinthe du monde) abondent en réflexions écologiques. Un aperçu de son engagement écologique, autre facette de son écologisme, est donné à la fin du volume Marguerite Yourcenar et l'écologie (1990), bulletin 2, Bruxelles, CIDMY. Pour ce qui est de l'intérêt que Yourcenar témoigna à la littérature dite écologique, voir WAGNER Walter (2008), Marguerite Yourcenar et Henry David Thoreau : un apprentissage écologique, Bulletin de la SIEY 29, Tours, SIEY, p. 65-82.

${ }^{4}$ SAnvitale Francesca (2002), Rencontre avec Marguerite Yourcenar (1986-1987), in : YouRCENAR Marguerite, Portrait d'une voix, op. cit., p. 355 sq.

${ }^{5}$ RESTORI Enrica (1994), Un anthropomorphisme à rebours : de la voix humaine à la voix des choses, in: Vazquez de Parga María José et Poignault Rémy (dir.), L'universalité dans l'œuvre de Marguerite Yourcenar. Actes du colloque international Tenerife (Espagne) novembre 1993, Tours, SIEY, p. 148.

${ }^{6}$ Desblache Lucile (1997), Marguerite Yourcenar et le monde animal. Éthique et esthétique de l'altérité, Bulletin de la SIEY 18, Tours, SIEY, p. 155.
} 
récit, tandis que Pascale Doré parle d'un Nathanaël « métamorphosé petit à petit en animal ${ }^{7}$. Marthe Peyroux, à son tour, remarque la place privilégiée du protagoniste au sein de l'univers écologique de l'auteur : « De tous les personnages yourcenariens, c'est le meilleur lecteur du monde naturel, en vérité c'est là un des rôles qui lui est imparti $»^{8}$.

Une fois posée l'évidence de la thématique écologique d'Un homme obscur, nous pouvons poser les questions suivantes : Comment ce texte traduit-il les rapports entre l'homme et son environnement naturel ? Comment cette fiction peint-elle le dualisme culture-nature, qui est typique de l'ontologie occidentale? Quelle philosophie écologique apparaît dans ce récit ${ }^{9}$ ?

Avant d'explorer ces pistes, résumons brièvement la diégèse d'Un homme obscur. Ce récit raconte l'histoire de Nathanaël, jeune homme vivant au XVII ${ }^{\mathrm{e}}$ siècle, qui, à la suite d'une rixe, est obligé de s'enfuir. Commence alors un périple à bord d'un bateau de son Angleterre natale en Hollande en passant par les Caraïbes et l'Amérique du Nord. De constitution physique fragile, il travaille dans l'imprimerie de son oncle à Amsterdam où il se marie avec la prostituée Saraï, qui finit par le quitter. Indigent et malade, il est recueilli par Monsieur Van Herzog dans la maison duquel il assure le service de valet. À l'invitation de son maître, Nathanaël se rend dans une île frisonne déserte pour garder la chasse de Monsieur. Le protagoniste, ayant connu quelques amours et un peu de tendresse, meurt seul au milieu d'une nature sauvage.

Pour analyser le roman, nous avons choisi une approche écocritique ${ }^{10}$ qui nous fournit les outils méthodologiques nécessaires pour décrire la problématique écologique qui lui est sous-jacente.

L'interrelation de l'homme et la nature, domaine par excellence de l'écocritique, est aussi le propre de l'écologie scientifique telle que l'a fondée l'Allemand Ernst Haeckel : «Par écologie nous entendons l'ensemble de la science étudiant les rapports de l'organisme avec le monde extérieur ambiant, parmi

\footnotetext{
${ }^{7}$ Dore Pascale (1999), Yourcenar ou le féminin insoutenable, Genève, Droz, p. 77.

${ }^{8}$ Peyroux Marthe (2006), Marguerite Yourcenar. Un regard sur le monde, Paris, Eurédit, p. 27.

${ }^{9}$ Le courant environnementaliste qui nous intéresse ici est la Deep Ecology ou écologie profonde, terme forgé par le philosophe norvégien Arne Naess. Les adhérents de cette approche écologique visent à supplanter l'anthropocentrisme moderne par une vision écocentrique et holistique du monde dans lequel l'homme figure comme un élément parmi d'autres dans la biosphère. Cette forme radicale d'environnementalisme postule l'interdépendance de l'homme et de la nature et favorise un égalitarianisme biosphérique accentuant la valeur intrinsèque de toutes les entités «naturelles » y compris les paysages. Pour une définition succincte des quatre principales «écoles » écologiques voir GARRAD Greg (2004), Ecocrititcism, Londres et New York, Routledge, p. 20-30. Une initiation à l'écologie profonde est proposée par DRENGSON Alan et INOUE Yuichi (dir., 1995), The Deep Ecology Movement. An Introductory Anthology, Berkeley, North Atlantic Books.

${ }^{10}$ L'écocritique, théorie littéraire née aux USA à la fin des années quatre-vingts, comprend «l'étude de la relation de l'humain et le non-humain à travers l'histoire culturelle humaine, entraînant une analyse critique du terme 'humain' lui-même» (GARRAD Greg, 2004, Ecocriticism, op. cit., p. 5). La recherche écocritique, quasiment inexistante en France, se révèle être particulièrement féconde aux USA et en Angleterre. Elle est à l'origine d'un discours (souvent politique) ayant produit des centaines de monographies et d'articles, des revues spécialisées, des colloques et une plateforme internationale de discussion appelée ASLE (Association for the Study of Literature and the Environment) avec une antenne en Europe.

(Nous traduisons toutes les citations anglaises et allemandes.)
} 
lesquels nous pouvons compter, par extension, toutes les 'conditions d'existence' ${ }^{11}$. Le biologiste ne s'intéressait évidemment qu'au monde animal, mais grâce à sa définition du grand réseau de relations dominant la vie sur notre planète, il a indirectement jeté les bases de notre conception moderne de l'environnement dont l'homo sapiens fait partie intégrante.

Les rapports qu'entretient Nathanaël avec la nature sont intenses et multiples. Capable d'une force d'identification hors du commun, il est sensible à toute forme de souffrance, humaine ou animale : «enfant ou vieillard, homme ou femme, animal ou bipède qui parle et travaille de ses mains, tous communiaient dans l'infortune et la douceur d'exister $»^{12}$.

Cette sympathie pour toutes les espèces lui interdit d' $"$ achever au couteau » ${ }^{13}$ le gibier attrapé dans des fosses, pratique courante dans l'Île Perdue, où il a échoué, l'incitant à protéger les animaux des mauvais traitements que leur font subir les hommes. Également en raison de cette attitude non-violente, il «préférait à la pêche le ramassage des baies $»^{14}$ et il renonce à manger de la viande. Foncièrement pacifique, il a le sentiment que jamais « il n'avait pas fait de mal, fût-ce seulement une pierre jetée à un oiseau, ou un mot cruel qui suppurerait dans la mémoire de quelqu'un $\gg$.

La pitié qu'il éprouve pour les animaux se transforme en charité lorsqu'il s'agit des humains, vertu mise en lumière au moment où il donne à boire à un Jésuite français agonisant après avoir été frappé par un marin anglais ${ }^{16}$ : «Nathanaël lui souleva la tête, et s'adressa à lui, d'abord en anglais, puis en néerlandais, sans se faire comprendre. Il s'avisa alors de lui demander en latin ce qu'il pouvait pour le soulager $\gg{ }^{17}$.

Solidaire de la création et observateur passionné de la vie sous toutes ses formes, le héros est «toujours tenté de chercher des ressemblances entre l'animal et l'homme $»^{18}$, et il finit même par voir la frontière s'estomper. Dans une perspective écocritique, nous pouvons dire que Nathanaël remet en question le dualisme culture-nature. La traditionnelle différence entre res cogitans et res extensa, pour parler comme Descartes, n'a plus droit de cité aux yeux de ce jeune homme à la fois «simple » et éclairé. La nature ne représente plus la matière sans esprit et émotions éternellement disponible à la satisfaction de nos besoins matériels et, par conséquent, à l'exploitation. Tout au contraire, à un même degré

\footnotetext{
11 HAECKEL Ernst (1988), Generelle Morphologie der Organismen, tome II (Allgemeine Entwicklungsgeschichte der Organismen), Berlin, Reimer, 1866. Réimpression Berlin, de Gruyter, p. 286.

${ }_{12}$ YourCENAR Marguerite (1982), Un homme obscur, Euvres romanesques, op. cit., p. 1036.

${ }^{13}$ Ibid., p. 957.

${ }^{14}$ Ibid.

${ }^{15}$ Ibid., p. 1035.

${ }^{16}$ Ce fait contredit le reproche de misanthropie, voire d'antihumanisme fréquemment adressé aux écologistes radicaux. Voir Bramwell Anna (1989), Ecology in the $20^{\text {th }}$ Century, New Haven et Londres, Yale University Press, p. 16 : «Les écologistes croient à l'harmonie essentielle de la nature. Mais c'est une harmonie à laquelle l'on doit peut-être sacrifier l'homme. » Même diagnostic négatif de la part d'un philosophe français constatant à l'égard des écologistes profonds une «haine de l'humain comme tel » (FERRY Luc, 1992, Le Nouvel Ordre écologique. L'arbre, l'animal et l'homme, Paris, Grasset, p. 33).

${ }^{17}$ YourCENAR Marguerite (1982), Un homme obscur, Euvres romanesques, op. cit., p. 952.

${ }^{18}$ Ibid., p. 1023.
} 
que nos semblables, la nature, altérité par excellence, mérite notre attention et sollicitude. Nathanaël transcende la vision comparatiste de l'éthologue afin d'accéder à un égalitarisme universel qui supprime toutes les barrières entre notre espèce et la nature animée ${ }^{19}$ : «Il ne se sentait pas, comme tant de gens, homme par opposition aux bêtes et aux arbres ; plutôt frère des unes et lointain cousin des autres $\gg{ }^{20}$.

Si la sensibilité et l'imagination facilitent l'identification avec les règnes animal et végétal, elles peuvent également réveiller ou stimuler des penchants animistes disparus de la civilisation. Le protagoniste fait preuve de cet atavisme lorsqu'il inspecte une plantation d'arbres qui a été rasée par une tempête: « Nathanaël espérait que ces vigoureux jeunes frères, serrés les uns contre les autres se seraient mutuellement protégés ${ }^{21} \gg$. En rapprochant les humains de tous les êtres vivants, le sujet anthropomorphise les objets, procédé courant dans les fables et contes de fées ainsi que dans toute Weltanschauung mythologique.

Même si certains contemporains s'identifient avec tous les êtres vivants et, par conséquent, les considèrent comme dignes de compassion, les humains et les animaux appartiennent à deux sphères ontologiques séparées. Or, tout en remettant en cause la supériorité humaine, Yourcenar, Nathanaël et les écologistes radicaux doivent reconnaître que malgré une capacité de jouissance et de souffrance qui est comparable, les différences entre les hommes et les bêtes restent nombreuses. N'oublions pas que ce sont les êtres humains qui sont dotés d'une sensibilité et d'une intelligence suffisamment complexes pour étudier la faune et s'y identifier. Seuls les humains possèdent une éthique et sont aptes à régler leur comportement sur une axiologie comptant la pitié parmi ses valeurs. Nathanaël s'aperçoit de cette faille fondamentale ne limitant pas seulement la communication entre les espèces mais l'aidant à comprendre que nos rapports avec les animaux seront toujours anthropocentriques. Autrement dit, l'homme n'est pas nécessaire à l'animal (sauf éventuellement les espèces domestiques) auxquels ne peuvent s'appliquer des notions morales. En observant les oiseaux sauvages dans l'île frisonne, le protagoniste d'Un homme obscur ne se trompe pas sur la solitude foncière de l'humain au sein de la création :

Nathanaël savait que rien de lui n'importait à ces âmes d'une autre espèce ; elles ne lui rendaient pas amour pour amour ; il eût pu les tuer, s'il avait senti en lui la moindre parcelle des

\footnotetext{
${ }^{19}$ Yourcenar va encore plus loin dans une tentative de supprimer la division entre la culture et la nature et réclame l'égalité de tous les êtres : «J'ignore pourquoi l'on parle toujours d'une humanité séparée du reste de la Créature ? Animaux, plantes et pierres, astres et aires ne font-ils pas eux aussi partie de l'humanité ?» (Yourcenar Marguerite, 1999, Sources II, Paris, Gallimard, p. 331). Voir aussi à ce propos la lettre à Odette Schwartz du 31 décembre 1977 et où elle s'oppose à toute forme de discrimination: "Enfin, égalité totale de tous les êtres humains sans distinction de sexe et de couleur. Et pourquoi pas égalité de tous les êtres sans distinction d'espèce? » (YourcenAR Marguerite, 1995, Lettres à ses amis et quelques autres, Paris, Gallimard, p. 581).

${ }^{20}$ YourCEnAR Marguerite (1982), Un homme obscur, CEuvres romanesques, op. cit., p. 1035. Croyant au «caractère arbitraire de la barrière entre les espèces » (SINGER Peter, 2002, Animal Liberation, New York, Harper Collins, p. XIII), l'auteur, à l'instar de Nathanaël, cherche des expériences zoophiles hors du commun : «Je n'aurai rien tant aimé que ces rencontres à travers le mur des espèces ; l'oiseau qui vous parle ou se pose sur votre main, l'écureuil pas trop effrayé, le chien amical » (YourCENAR Marguerite, 1999, Sources II, op. cit., p. 249).

${ }^{21}$ Ibid., p. 1040 sq.
} 
instincts du chasseur, mais non les aider dans leur existence exposée aux éléments et à l'homme. Les lapins dans l'herbe courte des dunes n'étaient pas non plus des amis, mais des visiteurs sur leurs gardes, sortis de leurs terriers comme d'un autre monde ${ }^{22}$.

La réflexion du héros surmonte le topos pastoral d'une communauté édénique où humains et animaux s'adonnant à la joie de vivre, n'ayant plus à endurer les privations d'une existence faite de luttes et de guerres. Même si le pacifisme de Nathanaël permet aux populations d'oiseaux de se propager tranquillement, elles ne peuvent pas non plus atténuer la solitude éprouvée par le chétif garde-chasse. Le regard que Nathanaël porte sur le monde naturel est celui d'un homme «éclairé », c'est-à-dire étonnement moderne, ne se trompant plus sur le rôle et la place de l'humain dans la biosphère. Conscient de notre lot d'hybride vivant au carrefour de la culture et de la nature, Nathanaël sait que pourtant le chemin de retour vers la nature sera à jamais barré ${ }^{23}$.

Il est intéressant, dans ce contexte, d'étudier le portrait des Indiens brossé par Yourcenar dans ce récit: du fait qu'ils vivent dans l'intimité de la nature, ils devraient parvenir à atteindre l'idéal d'une coexistence harmonieuse avec l'environnement, modèle à suivre selon les adeptes de l'écologie profonde. Arne Naess, fondateur de ce mouvement, souligne d'ailleurs que les écologistes étudient le mode de vie des peuples dits «primitifs » pour savoir s'ils parviennent ou parvinrent à maintenir l'équilibre écologique. L'écophilosophe cite les Indiens d'Amérique du Nord, concluant qu'effectivement ceux-ci font preuve d'une «relation écologiquement plus responsable entre les humains et le monde non humain ${ }^{24}$ que les sociétés postindustrielles.

Dans Un homme obscur, Nathanaël rencontre des Peaux-Rouges lorsque son bateau atteint la côte nord-américaine. Les indigènes s'approchant dans des

\footnotetext{
${ }^{22}$ Ibid., p. 1028.

Cette leçon en reflète une autre apprise par Yourcenar elle-même: «Vu aujourd'hui la sage grenouille sur le roc au bord du point d'eau du jardin. Immobile, comme minérale, buvant la lumière et l'air, très ancienne et vénérable créature douée d'une sagesse amphibie. Et si loin de moi qu'il n'existe aucun moyen pour lui faire percevoir l'amitié que j'ai pour elle» (YourCENAR Marguerite, 1999, Méditations dans un jardin, Sources II, op. cit., p. 247). Yourcenar, qui reconnaît les limites de communication entre deux consciences tout à fait différentes, à savoir celle de l'humain et celle de l'animal, contredit précisément cette constatation dans un entretien : «On peut d'ailleurs, quand on le veut, avoir pour amis des animaux, des plantes ou des pierres, et alors la réciprocité devient différente : les animaux, eux, nous aiment avec un affectueux égoïsme qui n'est pas si différent de celui de beaucoup de nos amis humains ; ils nous aiment (et c'est bien naturel) pour ce que nous leur donnons. Les plantes aussi pratiquent la réciprocité ; elles nous remercient de nos soins par la façon qu'elles ont de croître ou de fleurir. Et qui s'est adossé à un rocher pour se protéger du vent, qui s'est assis sur une pierre chauffée par le soleil, en y posant les mains pour essayer de capter ces obscures vibrations que nos sens ne perçoivent pas, a bien de la peine à ne pas croire obscurément à l'amitié des pierres » (Yourcenar Marguerite, 1997, Les yeux ouverts. Entretiens avec Matthieu Galey, Paris, Bayard Editions, p. 307).

${ }^{23}$ Le désir de retourner à la nature, caractéristique de l'homme moderne, découle du fait que nous portons l'empreinte indélébile de la culture humaine. Voir à ce propos Schiller : "Tant que nous étions de simples enfants de la nature, nous étions heureux et parfaits ; nous sommes devenus libres et nous avons perdu les deux. Il en résulte une nostalgie double et inégale de la nature ; une nostalgie de sa béatitude, une nostalgie de sa perfection» (SCHILLER Friedrich, 2002, Über naive und sentimentalische Dichtung, Stuttgart, Reclam, p. 23).

${ }^{24}$ NAESs Arne (2008), Cultural Diversity and the Deep Ecology Movement, The Ecology of Wisdom. Writings by Arne Naess, Berkeley, Counterpoint, p. 121.
} 
pirogues offrent aux matelots de la nourriture en échange d'alcool, n'hésitant pas à guider le capitaine dans les passages difficiles. Serviables, paisibles et passionnés de boissons fortes, les sauvages correspondent à l'image de l'homme naturel ignorant envie, avarice et violence, et le narrateur renforce cette impression favorable en faisant leur éloge : «Les Micmacs et les Abenakis qui fréquentaient l'île dans la saison de la pêche étaient sans malice envers ces quelques blancs tirant à grand-peine du sol leur maigre provende $»^{25}$.

Peu à peu, cependant, cette image s'entache, montrant des sauvages moins nobles qu'il ne parait. Nathanaël s'étonne qu'ils attribuent une valeur marchande exorbitante à certains objets fabriqués par des blancs et se plaint de leur manque d'hygiène ne les empêchant pas «de pisser droit devant eux, où qu'ils se trouvassent, même à l'intérieur des huttes $»^{26}$, comportement qu'on associerait plutôt aux bêtes. Non contents d'être belliqueux, ils sont aussi cruels, prenant du plaisir à assommer des animaux attrapés et infligeant des tortures atroces à leurs prisonniers. En apercevant les scalps dans leurs cabanes, Nathanaël doit penser aux têtes de suppliciés que l'on peut voir devant la Tour de Londres. D'où la conclusion désillusionnée qu'en matière éthique il n'y a aucune différence entre les Indiens et les Européens : «les hommes sont partout des hommes ${ }^{27}$.

Malgré le bilan mitigé des mœurs des sauvages, «l'engagement écologique de l'auteur montre le bout de l'oreille ${ }^{28}$ lorsque Nathanaël admire «leur soin de ne prélever sur le gibier que le strict nécessaire pour apaiser leur faim $»^{29}$. L'exploitation des ressources naturelles, l'une des causes du déséquilibre écologique dont la planète souffre actuellement, fut un problème ignoré des civilisations disparues ${ }^{30}$ et c'est justement cette sagesse écologique qui vaut la peine d'être adoptée si nous voulons freiner la destruction de l'environnement comme Yourcenar le déclare une fois de plus dans une interview : «Ne pas peser sur la terre. Tout est là » ${ }^{31}$.

Si les Indiens passent pour des écologistes avant la lettre, Nathanaël les surpasse sur le plan humain mais aussi dans son respect pour la nature car, à la différence des autochtones insulaires, il éprouve de la pitié pour les animaux, si bien qu'il essaie de les protéger des hommes. Sa bonté dépasse celle des sauvages qui, tout en considérant la nature comme sacrée, s'inscrivent dans une éthique plutôt utilitaire.

\footnotetext{
${ }^{25}$ YourCENAR Marguerite (1982), Un homme obscur, Euvres romanesques, op. cit., p. 958.

${ }^{26}$ Ibid., p. 959.

${ }^{27}$ Ibid.

${ }^{28}$ Pelckmans Paul (1993), Nathanaël au Canada. Un faux procès du Bon Sauvage, Nathanaël pour compagnon. Dix études sur «Un homme obscur» de Marguerite Yourcenar. Bulletin de la SIEY 12, Tours, SIEY, p. 50.

${ }^{29}$ YouRCENAR Marguerite (1982), Un homme obscur, CEuvres romanesques, op. cit., p. 959.

Voir aussi le jugement favorable que l'auteur exprime au sujet de l' «écologisme» des Indiens : «Les Anciens se trompaient comme nous. Ils condamnaient néanmoins ce qu'ils appelaient la 'démesure'. Les Indiens d'Amérique la redoutaient aussi, semblables en cela à la plupart des primitifs » (YouRCENAR Marguerite, 1997, Les yeux ouverts, op. cit., p. 282).

${ }^{30}$ L'exemple de l'Île de Pâques prouve qu'il y avait bien des exceptions.

${ }^{31}$ Pompon-Bailhache Pierrette (2002), L'Art de vivre de Marguerite Yourcenar. Une leçon de sagesse sous un toit de bois (1978-1979), YourCenar Marguerite, Portrait d'une voix, op. cit., p. 207.
} 
Sa vénération pour la nature correspond à un écologisme moderne combinant des aspects scientifiques, éthiques, spirituels et esthétiques et que nous exposerons plus loin. Si l'épistémologie écologique moderne se fonde sur l'idée d'interrelations des domaines de la culture et de la nature, c'est-à-dire d'un holisme biosphérique, où l'ensemble d'un écosystème dépasse la somme de ses parties, l'écocritique, comme nous l'avons vu, s'intéresse aux relations (souvent aliénées) entre l'humain et son environnement naturel. Elle met en relief nos attitudes politiques, morales, émotionnelles ou spirituelles, donc tout le côté non quantifiable de nos rapports avec la nature, et cherche à redéfinir la place de l'homme dans le monde. Le contact que Nathanaël ne cesse d'établir avec l'environnement est dominé par des motifs purement émotionnels, spirituels et éthiques. Rappelons l'émerveillement, l'admiration et l'émotion que suscite le rendez-vous avec les phénomènes de la nature. C'est avec joie qu'il contemple un ours mangeant des framboises ou un renardeau dans une clairière. Mais ce n'est pas tout : «Le garçon chérissait de même les arbres ; il les plaignait, si grands et si majestueux qu'ils fussent, d'être incapables de fuir ou de se défendre, livrés à la hache du plus chétif bûcheron $»^{32}$.

À lire cette description isolée de son contexte, on penserait aux sensibleries d'un sentimental surmontant son malaise en société en attachant toute son affection aux animaux. Or, la plénitude de l'expérience humaine de Nathanaël confirme le contraire. Homme parmi les hommes, il parvient pourtant à vivre en parfaite harmonie avec une nature qu'il «comprend », ou plutôt ressent plus intensément que n'importe lequel de ses contemporains. Ayant un penchant pour la contemplation, il se transforme en mystique face à la beauté ineffable d'une nuit en pleine mer, se sentant «vivant, respirant, placé tout au centre ». ${ }^{33} \mathrm{Ce}$ que l'écologiste exprime avec des mots plus prosaïques, Nathanaël, complice des éléments et du cosmos, le ressent : « le Terrien est chevillé à la Terre », ${ }^{34}$ c'est-àdire notre existence physique mais aussi notre bien-être émotionnel (et parfois même mental) dépendent de notre environnement naturel. Conformément à cette logique, il s'allonge dans les dunes pour mourir: «On était bien là ». ${ }^{35}$ Le commentaire du narrateur omniscient met en relief le symbolisme de ce geste: finissant ses jours dans une île déserte et peuplée de colonies d'oiseaux, Nathanaël nous fait savoir où se trouve sa vraie patrie. Entouré d'arbustes et bercé par le coassement des oiseaux, l'homme fraternisant avec les animaux et les arbres

\footnotetext{
${ }^{32}$ YourCEnAR Marguerite (1982), Un homme obscur, Euvres romanesques, op. cit., p. 958.

L'amour des arbres se fait sentir tout au long de l'œuvre yourcenarienne. Le motif de l'arbre exposé à la violence du bûcheron se trouve déjà dans L'Euvre au Noir où des charbonniers sont traités de «bourreaux des arbres » (Yourcenar Marguerite, 1982, L'Euvre au Noir, Euvres romanesques, op. cit., p. 585). Voir aussi l'éloge des arbres dans un fragment intitulé «Écrit dans un jardin » : [...] sursaut de révolte en présence du bûcheron et l'horreur, mille fois plus grande, devant la scie mécanique. Abattre et tuer ce qui ne peut pas fuir » (YourCENAR Marguerite, 1991, Le Temps, ce grand sculpteur, in: Yourcenar Marguerite, Essais et mémoires, Paris, Gallimard, coll. de la Pléiade, p. 405).

${ }^{33}$ Ibid., p. 962.

${ }^{34}$ PaQuOT Thierry (2007), Petit manifeste pour une écologie existentielle, Paris, Bourin Editeur, p. 35 .

${ }^{35}$ YourCenAR Marguerite (1982), Un homme obscur, Euvres romanesques, op. cit., p. 1041.
} 
comme pour supprimer définitivement le dualisme culture-nature, (re)devient « chose parmi les choses ${ }^{36}$.

Après ce tour d'horizon de l'éthique écologique de Nathanaël, il est temps de se demander quel courant écologique il représente, car même si la fiction se déroule au XVII ${ }^{\mathrm{e}}$ siècle, le protagoniste est imprégné par les concepts écologiques formés au $\mathrm{XX}^{\mathrm{e}}$ siècle. Parmi les quatre courants environnementaux évoqués au début de notre article, l'écologie profonde nous semble le mieux illustrer le mode de vie exemplaire de cet Homme obscur. Cette écosophie ${ }^{37}$ se résume par une éthique fondée sur huit principes fondamentaux formulés par les philosophes Arne Naess et George Sessions en 1984.

Le premier de ces axiomes s'applique parfaitement au message du récit de Yourcenar : «1. Le bien-être et l'épanouissement des formes de vie humaines et non-humaines de la Terre ont une valeur en elle-même (synonyme: valeur intrinsèque, valeur inhérente). Ces valeurs sont indépendantes de l'utilité du monde non-humain pour les besoins humains ${ }^{38}$. Implicitement, Nathanaël reconnaît ce postulat sans lequel la sympathie et la pitié qu'il éprouve pour les animaux et les arbres seraient absurdes. De plus, jamais il ne juge le monde naturel selon son utilité et se contente d'adopter une attitude purement admirative et contemplative à l'égard des êtres non humains.

Le troisième principe se trouve également mis en pratique par ce précurseur écologique : «3. L'Homme n'a pas le droit de réduire la richesse et la diversité biologique, sauf pour satisfaire des besoins humains vitaux ${ }^{39}$. Comme nous l'avons vu, le héros du récit yourcenarien, loin d'intervenir ou de porter atteinte à son environnement, défend le droit de vivre des animaux.

Afin de réaliser une diminution de l'impact des activités humaines sur la biosphère et une extension de la vie sauvage sur notre planète, l'écologie profonde préconise un changement des paradigmes culturels de la société de consommation : «7. Les changements idéologiques passent par l'appréciation d'une bonne qualité de vie plutôt que l'adhésion à des standards de vie toujours plus élevés. Il faut prendre conscience de la différence entre 'bonne qualité' et 'course au niveau de vie extrêmement élevé' (qui serait néfaste à la nature) ». ${ }^{40}$ Malgré l'anachronisme de cette règle dans une fiction située au XVII ${ }^{\mathrm{e}}$ siècle, elle s'applique parfaitement à Nathanaël qui se distingue de ses contemporains dans la mesure où, intuitivement, il met la perfection personnelle au-dessus du matérialisme omniprésent. Son empathie et son humanisme lui inspirent un respect profond pour tous les êtres vivants, l'emportant sur la bienveillance intéressée et superficielle de Monsieur Van Herzog se servant aussi de son jeune valet pour amuser ses amis érudits. On se rappellera la curiosité avec laquelle ils demandent à Nathanaël toutes sortes de renseignements et de détails (parfois grivois) sur la vie des indigènes dans les îles

\footnotetext{
${ }^{36}$ Ibid., p. 1033.

37 Pour la définition voir Naess : «Par écosophie je comprends une philosophie d'harmonie ou d'équilibre écologique» (NAESS Arne, 1995, The Shallow and the Deep, Long-Range Ecology Movement, in : Drengson Alan et Inoue Yuichi (dir.), The Deep Ecology Movement, op. cit., p. 8).

${ }^{38}$ NAESS Arne et SESSIONS George (1995), Platform Principles of the Deep Ecology Movement, in : Drengson Alan InOuE et Yuichi (dir.), The Deep Ecology Movement, op. cit., p. 49.

${ }^{39}$ Ibid., p. 49.

${ }^{40}$ Ibid., p. 50.
} 
qu'il a visitées. Cet homme si modeste a acquis une sagesse grâce à laquelle il parvient à comprendre la dimension écologique de la condition humaine. Parent lointain des bêtes et des plantes, il souscrit à un holisme gommant la frontière soigneusement gardée par les adeptes d'un anthropocentrisme intégriste. L'écosophie pratiquée par Nathanaël ne reflète pas uniquement l'utopie écologique telle que la rêva Yourcenar, mais aussi l'esprit de l'écologie profonde dont les huit principes fondamentaux ne sont pas entièrement applicables au protagoniste d'Un homme obscur dans la mesure où ils ont été conçus en vue des préoccupations et besoins d'une époque différente.

Parmi les convergences avec l'écologie profonde figure le concept de «moi écologique » ou ecological self ${ }^{41}$ qui rompt le cadre restreint de l'ego et que Naess définit ainsi : "Le moi écologique d'une personne est tout ce à quoi cette personne s'identifie $»^{42}$. Une telle «personnalité » engloberait idéalement la biosphère et mènerait tout naturellement à une éthique environnementale selon laquelle toute intervention dans le monde constituerait une atteinte portée à notre personne. Si l'avantage quant à la conservation du milieu naturel paraît évident, il risque, à la limite, de charger l'individu d'une responsabilité difficile à assumer. Nous n'envisagerons pas non plus ici les implications philosophiques d'un moi écologique qui, du fait de son extension, se transformerait en «super-ego » anthropocentrique qui absorberait l'ensemble de la nature et de la culture.

Finalement nous rappelons le pacifisme du protagoniste d'Un homme obscur et auquel souscrivent les adeptes de l'écologie profonde mais qui ne constitue pas nécessairement un dogme dans d'autres branches de l'environnementalisme comme le prouvent par exemple les actions controversées du $\mathrm{WWF}^{43}$.

Au terme de notre étude, nous arrivons à une double conclusion. Compte tenu de l'importance de l'actant «nature » dans la diégèse et de l'ampleur de la réflexion environnementale du protagoniste, nous croyons justifié de qualifier de «testament écologique » la dernière fiction de Yourcenar ${ }^{44}$. Par ailleurs, le dernier récit de Yourcenar est empreint d'un écocentrisme radical: son protagoniste défend l'égalité foncière entre l'humain et le règne animal. Son écologisme préfigure les bases théoriques de l'écologie profonde, qui réclame la diminution de l'action humaine sur notre planète et stigmatise un antagonisme aberrant entre la culture et la nature. Par conséquent, c'est en réconciliant ces termes, seulement à

\footnotetext{
${ }^{41}$ L'analogie du moi écologique avec la compassion cosmique des bouddhistes s'impose mais ne peut être approfondie dans le cadre de ce travail. Pour l'influence du bouddhisme sur l'écologie profonde voir CoOPER David E. et JAMEs Simon P. (2005), Buddhism, Virtue and Environment, Aldershot et Burlington, Ashgate.

${ }^{42}$ NAESS Arne (1995), Self-Realization : An Ecological Approach to Being in the World, in : DREngson Alan et Inoue Yuichi (dir.), The Deep Ecology Movement, op. cit., p. 15.

${ }^{43}$ Un aperçu des écogestes préconisés par l'écologie profonde nous présente une fois de plus son fondateur : NAESS Arne (2008), Lifestyle Trends Within the Deep Ecology Movement, The Ecology of Wisdom. Writings by Arne Naess, op. cit., p. 140 sq.

${ }^{44}$ À titre d'information, précisons que la dernière conférence prononcée par Yourcenar quelques semaines avant sa mort portait sur son sujet préféré et aurait par là même une valeur encore plus testamentaire. Voir YouRCENAR Marguerite (1988), «...Si nous voulons encore essayer de sauver la terre », in : DuPLE Nicole (dir.), Le droit à la qualité de l'environnement : un droit en devenir, un droit à définir: actes de la $V^{e}$ Conférence internationale de droit constitutionnel, Québec, Editions Québec/Amérique, p. 21-33.
} 
première vue contradictoires, que l'équilibre écologique aurait la chance d'être rétabli. Nathanaël, dans cette optique, ferait figure de précurseur.

\section{BIBLIOGRAPHIE}

\section{TEXTES}

YourCENAR Marguerite (1982), L'Euvre au Noir, Euvres romanesques, Paris, Gallimard, coll. de la Pléiade.

YOURCENAR Marguerite (1982), Postface d'Un homme obscur, Euvres romanesques, Paris, Gallimard, coll. de la Pléiade, p. 1065-1070.

YouRCENAR Marguerite (1982), Un homme obscur, Euvres romanesques, Paris, Gallimard, coll. de la Pléiade.

YOURCENAR Marguerite (1988), «...Si nous voulons encore essayer de sauver la terre », in : DUPLE Nicole (dir.), Le droit à la qualité de l'environnement : un droit en devenir, un droit à définir : actes de la $V^{e}$ Conférence internationale de droit constitutionnel, Québec, Editions Québec/Amérique, p. 21-33.

YourCENAR Marguerite (1991), Le Temps, ce grand sculpteur, in : YouRCENAR Marguerite, Essais et mémoires, Paris, Gallimard, coll. de la Pléiade.

YOURCENAR Marguerite (1995), Lettres à ses amis et quelques autres, Paris, Gallimard.

YouRCENAR Marguerite (1997), Les yeux ouverts. Entretiens avec Matthieu Galey, Paris, Bayard Editions.

YOURCENAR Marguerite (1999), Sources II, Paris, Gallimard.

\section{LITTERATURE SECONDAIRE}

BRAmWEll Anna (1989), Ecology in the $20^{\text {th }}$ Century, New Haven et Londres, Yale University Press.

COOPER David E. et JAMES Simon P. (2005), Buddhism, Virtue and Environment, Aldershot et Burlington, Ashgate.

DESBLACHE Lucile (1997), Marguerite Yourcenar et le monde animal. Éthique et esthétique de l'altérité, Bulletin de la SIEY 18, Tours, SIEY, p. 143-156.

DORE Pascale (1999), Yourcenar ou le féminin insoutenable, Genève, Droz.

DRENGSON Alan et INOUE Yuichi (dir., 1995), The Deep Ecology Movement. An Introductory Anthology, Berkeley, North Atlantic Books.

FERRY Luc (1992), Le Nouvel Ordre écologique. L'arbre, l'animal et l'homme, Paris, Grasset.

GARRAD Greg (2004), Ecocriticism, Londres et New York, Routledge.

HAECKEL Ernst (1988), Generelle Morphologie der Organismen, tome II (Allgemeine Entwicklungsgeschichte der Organismen), Berlin, Reimer, 1866. Réimpression Berlin, de Gruyter.

Marguerite Yourcenar et l'écologie (1990), bulletin 2, Bruxelles, CIDMY.

NAESS Arne (1995), Self-Realization : An Ecological Approach to Being in the World, in : DREnGSON Alan et InOuE Yuichi (dir.), The Deep Ecology 
Movement. An Introductory Anthology, Berkeley, North Atlantic Books, p. 13-30.

NAESS Arne (1995), The Shallow and the Deep, Long-Range Ecology Movement, in : Drengson Alan et InOUE Yuichi (dir.), The Deep Ecology Movement. An Introductory Anthology, Berkeley, North Atlantic Books, p. 3-9.

NAESS Arne (2008), Cultural Diversity and the Deep Ecology Movement, The Ecology of Wisdom. Writings by Arne Naess, Berkeley, Counterpoint, p. 120122.

NAESS Arne (2008), Lifestyle Trends Within the Deep Ecology Movement, The Ecology of Wisdom. Writings by Arne Naess, Berkeley, Counterpoint, p. 140 sq.

NAESS Arne et SESSIONS George (1995), Platform Principles of the Deep Ecology Movement, in : DREnGSON Alan, InOUE et YuICHI (dir.), The Deep Ecology Movement. An Introductory Anthology, Berkeley, North Atlantic Books, p. 4953.

PAQUOT Thierry (2007), Petit manifeste pour une écologie existentielle, Paris, Bourin Editeur.

PelCKMANS Paul (1993), Nathanaël au Canada. Un faux procès du Bon Sauvage, Nathanaël pour compagnon. Dix études sur «Un homme obscur» de Marguerite Yourcenar. Bulletin de la SIEY 12, Tours, SIEY, p. 45-56.

PEYROUX Marthe (2006), Marguerite Yourcenar. Un regard sur le monde, Paris, Eurédit.

POMPON-BAILHACHE Pierrette (2002), L'Art de vivre de Marguerite Yourcenar. Une leçon de sagesse sous un toit de bois (1978-1979), in : YOURCENAR Marguerite, Portrait d'une voix. Vingt-trois entretiens (1952-1987), Paris, Gallimard, p. 201-219.

RESTORI Enrica (1994), Un anthropomorphisme à rebours : de la voix humaine à la voix des choses, in: VAZQuEZ DE PARgA María José et PoIGnAult Rémy (dir.), L'universalité dans l'œuvre de Marguerite Yourcenar. Actes du colloque international Tenerife (Espagne) novembre 1993, Tours, SIEY, p. 137-151.

SANVITALE Francesca (2002), Rencontre avec Marguerite Yourcenar (1986-1987), in : YourCENAR Marguerite, Portrait d'une voix. Vingt-trois entretiens (19521987), Paris, Gallimard, p. 353-377.

SAVIGNEAU Josyane (2002), La Bienveillance singulière de Marguerite Yourcenar (1984), in : YourcenAR Marguerite, Portrait d'une voix.), Paris, Gallimard, p. 311-328.

SCHILLER Friedrich (2002), Über naive und sentimentalische Dichtung, Stuttgart, Reclam.

SINGER Peter (2002), Animal Liberation, New York, Harper Collins.

WAGNER Walter (2008), Marguerite Yourcenar et Henry David Thoreau : un apprentissage écologique, Bulletin de la SIEY 29, Tours, SIEY, p. 65-82. 


\section{SUMMARY}

This paper seeks to prove that Yourcenar's last fiction, the short novel $U n$ homme obscur, anticipates the theories and values of the Deep Ecology Movement, whose founder was the Norwegian philosopher Arne Naess. His so-called ecosophy is meant to overcome the hegemonial relationship between man and his natural environment by stressing the intrinsic value of all non-human nature and abolishing its utilitarian status. As we have shown, Nathanaël, the hero of Yourcenar's novel, epitomizes the basic principles of Deep Ecology and does not only reveal the writer's environmental consciousness but also confirms her role as an ecological forerunner in $20^{\text {th }}$ century French literature. 